University of Nebraska - Lincoln

DigitalCommons@University of Nebraska - Lincoln

Architectural Engineering -- Faculty Publications

Architectural Engineering and Construction,

Durham School of

2011

\title{
Non-Intrusive Electrical Load Monitoring and Profiling Methods for Applications in Energy Management Systems
}

\author{
Mahmoud Alahmad \\ University of Nebraska - Lincoln, malahmad2@unl.edu \\ Hosen Hasna $\mathrm{HH}$ \\ University of Nebraska-Lincoln, hhasna@unomaha.edu \\ Evans Sordiashie \\ University of Nebraska-Lincoln, sord.evans@huskers.unl.edu
}

Follow this and additional works at: https://digitalcommons.unl.edu/archengfacpub

Part of the Architectural Engineering Commons

Alahmad, Mahmoud; Hasna, Hosen HH; and Sordiashie, Evans, "Non-Intrusive Electrical Load Monitoring and Profiling Methods for Applications in Energy Management Systems" (2011). Architectural Engineering -- Faculty Publications. 49.

https://digitalcommons.unl.edu/archengfacpub/49

This Article is brought to you for free and open access by the Architectural Engineering and Construction, Durham School of at DigitalCommons@University of Nebraska - Lincoln. It has been accepted for inclusion in Architectural Engineering -- Faculty Publications by an authorized administrator of DigitalCommons@University of Nebraska Lincoln. 


\section{Non-Intrusive Electrical Load Monitoring and Profiling Methods for Applications in Energy Management Systems}

\author{
Mahmoud Alahmad, PhD, P.E \\ Durham School of Architectural \\ Engineering and Construction \\ University of Nebraska-Lincoln \\ Omaha, Nebraska \\ malahmad2@unl.edu
}

\author{
Hosen Hasna, MS Student \\ Durham School of Architectural \\ Engineering and Construction \\ University of Nebraska-Lincoln \\ Omaha, Nebraska \\ hhasna@unomaha.edu
}

\author{
Evans Sordiashie MS Student \\ Durham School of Architectural \\ Engineering and Construction \\ University of Nebraska-Lincoln \\ Omaha, Nebraska \\ Sord.evans@huskers.unl.edu
}

\begin{abstract}
Phantom Loads" cause energy waste in homes and the built environment as a whole. Consumers spend more than $\$ 3$ billion a year on "Phantom Load" in the United States alone. The goal of this paper is to conserve energy by increasing consumer awareness on their energy usage and reducing and /or eliminating phantom loads in the built environment. A further goal is to improve upon existing power distribution systems in the built environment with limited hardware additions to increase energy conservation. This paper investigates remote identification of load types and the locations along the electrical circuitry where they (load) are being consumed. The load type and status (on, off, standby) are determined both remotely and in a non-intrusive manner using Non-Intrusive Load Monitoring Methods. Time/Frequency Domain Reflectometry (TDR) is also being investigated to remotely locate energy consumption nodes along the electrical circuitry.
\end{abstract}

Keywords-Power distibution, Phantom Loads, Time Domain Reflectometry, Non-Intrusive Load Monitoring.

\section{INTRODUCTION}

The electric power sector is experiencing a major shift in its generation, transmission and distribution system, in an effort to reduce $\mathrm{CO}_{2}$ emissions, increase the use of renewable sources for a sustainable energy future and conserve precious resources by reducing energy consumption. The distribution system particularly plays a role in the built environment as it is an enabling product, an intangible necessity used to power our systems. The 2010 Annual Energy Outlook indicates a 14\% increase in U.S. primary energy consumption from 2008 to 2035 , an average annual growth rate of $0.5 \%$. Further, $\mathrm{CO}_{2}$ emissions are projected to increase by $0.3 \%$ per year for the same time frame. This increase is mostly due to the electric power and transportation sectors. According to United States Green Building Council (USGBC), the built environment in the United States accounts for $72 \%$ of electricity consumption, $39 \%$ of energy use, $38 \%$ of all $\mathrm{CO}_{2}$ emissions, $40 \%$ of raw materials use, $30 \%$ of waste output (136 million tons annually), and $14 \%$ of potable water consumption[2]. Electricity consumed by an electronic device while it is turned off or in standby mode is known as "Phantom Load". Focus on Energy with Wisconsin Utilities estimates more than $\$ 3$ billion dollars spent annually on phantom loads [3].

Active research into energy conservation in the built environment is focusing on more efficient and sustainable systems and energy consumption awareness and behavior change. This behavior change is more prominent in the residential sector with Advanced Metering Initiatives and Infrastructure (AMI) being developed to empower the user and to facilitate the future of the smart grid [4]. Most of the research is addressing the energy consumption from a top approach, i.e. looking at total consumption to influence behavior. A 2010 study by American Council for an EnergyEfficient Economy (ACEEE) of 57 energy conservation projects from 1970-2010 found that feedback devices alone are unlikely to maximize energy savings [5]. Hence, there is a need to focus on creative and promising methods to encourage energy efficient lifestyles. For example, identification of specific load and location has recently emerged to develop smart home/building to conserve energy and provide for comfortable living and working environment.

In the process of creating a smart built environment, we are proposing to address energy needs using a bottom-up approach. The focus on this research will be on consumption and conservation at the electrical node, where an electrical node is a point on the electrical wiring system at which electrical current is taken to supply utilization equipment (load). Investigations are being done to develop non-intrusive and novel methods to identify the location of each node and the type and status of the connected loads. Information from these investigations will be used for modeling, simulation, and analysis to enable innovations in building information modeling (BIM), energy consumption performance, and energy conservation and recovery using impulse saving behavior at the residential level and demand side energy management at the commercial/utility level. Some emerging technologies employ a lot of hardware additions to existing circuitry to achieve the intended goal of this research. The ultimate goal of this research is to be able to identify and locate loads with minimal hardware additions to existing electrical circuitry in the built environment. Therefore, identification of location of loads and load types are being done 
remotely at the panel level. Identification of load points or location is being investigated through Time Domain Reflectometry (TDR) technology and load type is done through Non-Intrusive Load Monitoring Methods (NILM).

Fig.1 shows a block diagram of the proposed Non-Intrusive Electrical Load Monitoring System.

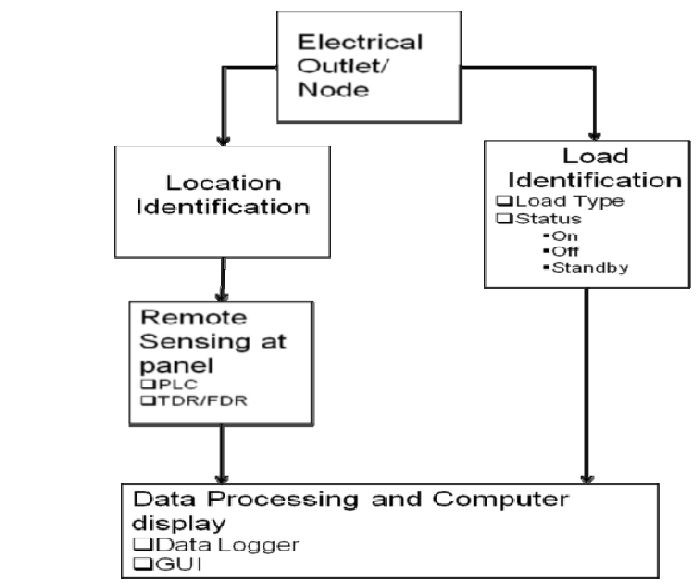

Figure 1, Block diagram of the Proposed Non-Intrusive Electrical Load Monitoring System.

\section{LOAD LOCATION IDENTIFICATION}

Time Domain or Frequency Reflectometry (TDR/FDR) is used to locate faults in all types of metallic paired cables. It is used to locate cabling problems such as sheath faults, broken conductors, water damage, loose connectors, shorted conductors, smashed cables, crimps, system components and a variety of cable faults. Time Domain or Frequency [6] Reflectometer technology employs an apparatus to send a shaped pulse wave along the current carrying conductor (electrical wire). The wave will reflect back if there is an open circuit along the current carrying conductor or no reflection occurs if an equipment or load is utilizing energy along the conductor which has impedance characteristics equal to that of the conductor. The propagation of the wave along the conductor depends on the Velocity of Propagation (VOP) or Velocity Factor of the conductor medium. Where velocity factor (VF) of the conductor is the speed at which the wave travels through the conductor, relative to the speed of light. In the event that reflection occurs, the difference in time measurement of when the pulse is sent and reflected back is recorded. This difference in time is used with the velocity factor (VF) of the cable to determine the distance at which the pulse was reflected back. The TDR/FDR technology is currently being investigated to locate and identify power outlet or node. A miniature version of the TDR/FDR apparatus will be used in the electric panel board to generate and send a wave pulse signal along the power distribution conductor linking the power outlets or nodes. When an outlet or node is not in use, reflected pulse signal display on TDR/FDR apparatus will only display node location (distance wise) along the electrical conductor. In the event that a particular outlet is in use at any time, the TDR/FDR will display the location of the node with some additional jump in the reflectometry signal due to impedance change at that location. The first phase of this investigation is to determine the location of all electrical nodes along a non-energized electrical conductor. Successful identification of nodes will be followed by analysis of node location on energized conductor using Spread Spectrum Time Domain Reflectometry (SS TDR). Figure 2 shows the proposed model of the Non-Intrusive Load monitoring system.

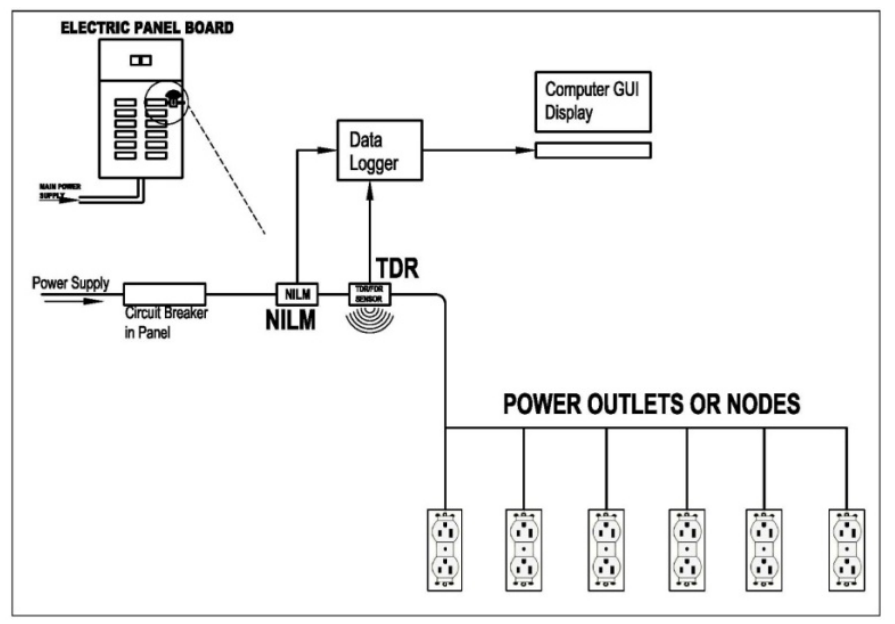

Figure 2, Proposed Model of the Non-Intrusive Load Monitoring system.

\section{A. Time Domain Reflectometry}

The principle of time domain reflectometer is illustrated in fig. 3. A pulse signal $E_{0}^{i}$ is sent into the conductor segment $\left(Z_{1}\right)$, part of the signal is reflected $\left(E_{0}^{r}\right)$ back at the boundary 0 and a transmitted component $\left(E_{0}^{t}\right)$ propagates into the second segment $\left(Z_{2}\right)$.

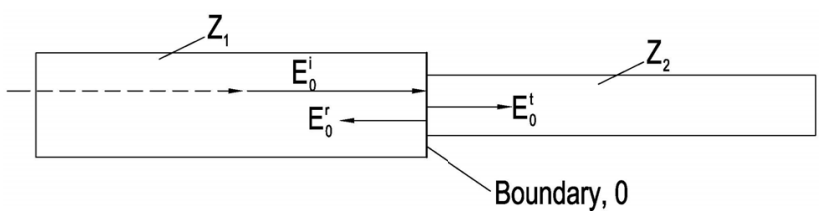

Figure 3, Transmission Line Analogue configuration for TDR.

The transmission line configuration shown in fig. 3, [7] the incident $\mathrm{E}_{0}^{\mathrm{i}}$, reflected $E_{0}^{r}$ and transmitted $E_{0}^{t}$ electric signal at the boundary 0 are related by:

$$
\begin{gathered}
E_{0}^{i}+E_{0}^{r}=E_{0}^{t} \\
\frac{E_{0}^{i}}{Z_{1}}+\frac{E_{0}^{r}}{Z_{1}}=\frac{E_{0}^{t}}{Z_{2}}
\end{gathered}
$$

Where $Z_{1}$, and $Z_{2}$ are the characteristic impedance of the transmission lines 1 and 2 respectively. Combining equations (1), (2) and eliminating $E_{0}^{t}$,

$$
E_{0}^{r}=\left(\frac{Z_{2}-Z_{1}}{Z_{2}+Z_{1}}\right) E_{0}^{i}, \quad E_{0}^{r}=\Gamma E_{0}^{i},
$$

Where $\Gamma$ is the reflection coefficient.

$$
\Gamma=\frac{E_{0}^{r}}{E_{0}^{i}}=\left(\frac{Z_{2}-Z_{1}}{Z_{2}+Z_{1}}\right)
$$

Dividing through (1), by $E_{0}^{i}$ 


$$
\begin{aligned}
& 1+\frac{E_{0}^{r}}{E_{0}^{i}}=\frac{E_{0}^{t}}{E_{0}^{i}}, \\
& 1+\Gamma=\tau
\end{aligned}
$$

Where $\tau$, is the transmission coefficient;

$$
\tau=1+\Gamma, \quad \tau=\frac{2 \mathrm{Z}_{2}}{\mathrm{Z}_{2}+\mathrm{Z}_{1}}
$$

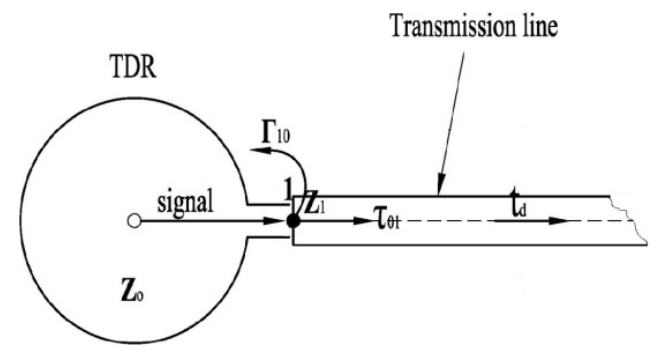

Figure 4, TDR signal on a transmission line.

Applying equations (3b) and (4b) to a TDR and a transmission line shown in fig. 4 , where the voltage signal is generated by the TDR,

$$
\begin{gathered}
\tau_{01}=1+\Gamma_{10} \\
\Gamma_{10}=\frac{Z_{1}-Z_{0}}{Z_{1}+Z_{0}},
\end{gathered}
$$

Where;

$\tau_{01}$ Is the transmitted signal from transmission line 0 to 1 .

$\Gamma_{10}$ Is the reflected signal from 1 to 0 .

$\mathrm{Z}_{0}, \mathrm{Z}_{1}$ are the characteristic impedance of the TDR and line 1 respectively.

$T_{d}$ is the travel time or delay for transmitted and reflected signal. $T_{d}$ is dependent on the velocity of propagation (VOP) of the conducting medium and is the same for transmission and reflection on the transmission line.

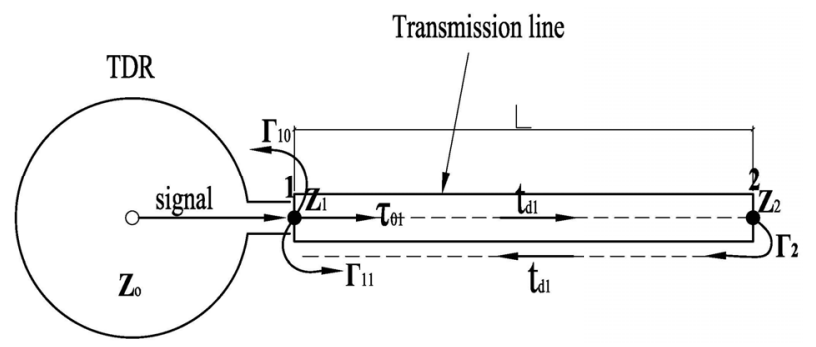

Figure 5, TDR - transmission line segment configuration.

For a TDR - transmission line segment show in fig. 5;

$\tau_{01}$ is the transmitted signal from point $0-1$, travelling to point 2 with $\mathrm{T}_{\mathrm{d}}=\mathrm{T}_{\mathrm{d} 1}$

$\tau_{10}$ is the transmitted signal from point $1-0$ back to TDR with $\mathrm{T}_{\mathrm{d}}=0$

$\Gamma_{10}$ is the reflected signal from point 1 to 0
$\Gamma_{11}$ is the reflected signal from point 1 back to the transmission line

$\Gamma_{2}$ is the reflected signal from point 2 , travelling back to TDR with $\mathrm{T}_{\mathrm{d}}=\mathrm{T}_{\mathrm{d} 1}$

$Z_{0}, Z_{1}, Z_{2}$ are the impedance of TDR and the characteristic impedance per length at point 1 and 2 respectively.

\section{B. TDR Preliminary Simulations and Results}

Preliminary simulations of the TDR investigation are limited to a non-energized electric circuit. Final simulations on energized electric conductor will later be investigated using Spread Spectrum Time Domain Reflectometry (SS TDR). Fig. 6 shows sample node locations of electrical circuitry. The conductor is a coated \# 14 of characteristic impedance 0.0104 $\mathrm{ohm} / \mathrm{m}$ and a VOP (VF) of 0.67 .

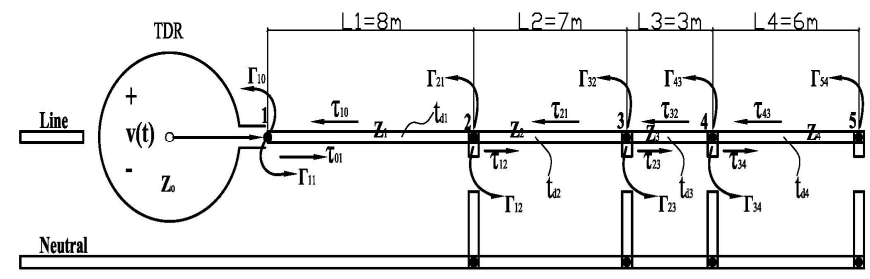

Figure 6, \#4 conductor with characteristic impedance of $0.0104 \mathrm{ohm} / \mathrm{m}$ and VOP/VF of 0.67 .

Table 1, TDR - configuration data for the setup in figure 6.

\begin{tabular}{|c|c|c|c|}
\hline Segment & Length/m & $\begin{array}{l}\text { Cumulative length } \\
\text { from TDR/m }\end{array}$ & Total Impedance/ $\mathbf{~}$ \\
\hline 1 & 8 & 8 & 0.0832 \\
\hline 2 & 7 & 15 & 0.1560 \\
\hline 3 & 3 & 18 & 0.1872 \\
\hline 4 & 6 & 24 & 0.2496 \\
\hline
\end{tabular}

Since the velocity of propagation is the same for each segment, normalization to the speed of light will be 1 . Therefore the propagation delay $\mathrm{T}_{\mathrm{d}}$ is given by;

$$
T_{d}=\frac{1}{2} \times(\text { length of segement }) \times \frac{0.67}{0.67}
$$

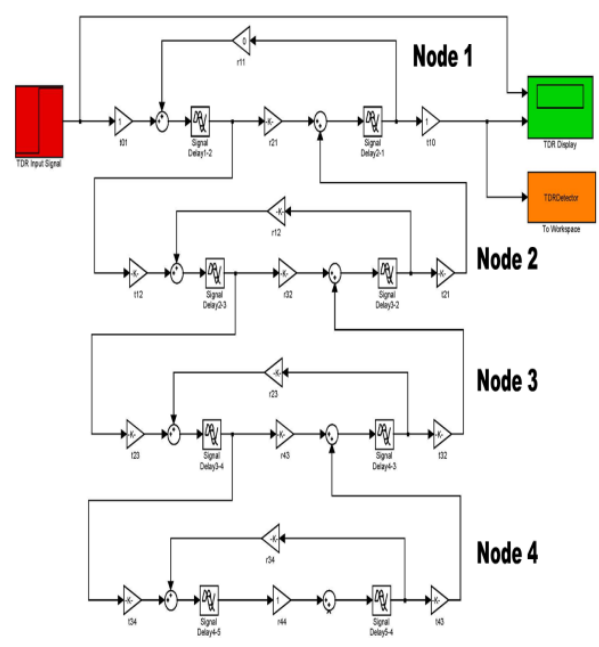

Fig.7, Simulink Block diagram of TDR setup in fig.6.

Durham School of Architectural Engineering- UNL 


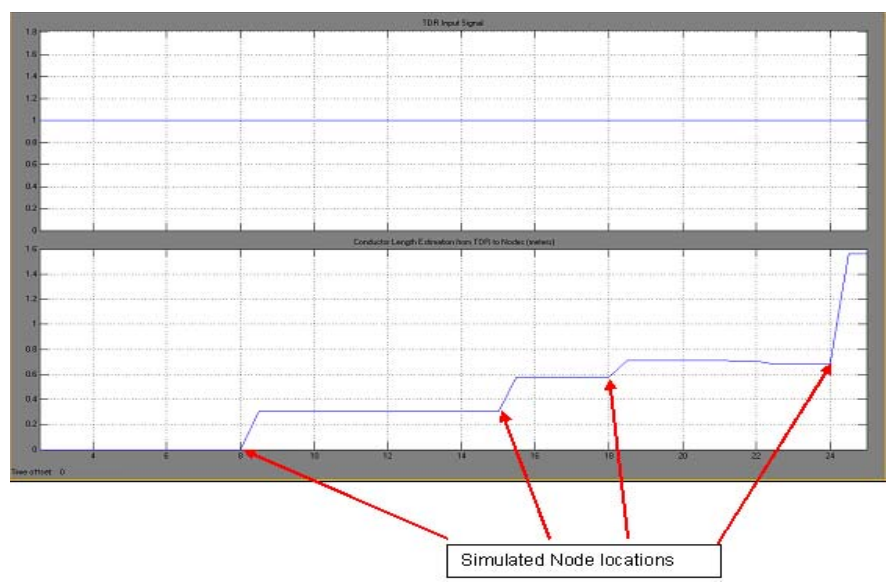

Figure 8, Simulated TDR display of nodes shown in fig. 6 .

From the simulated results, the reflected signals by the TDR show a jump in signal amplitude from segment to segment along the conductor. The various node locations are shown on the graph as $8,15,18$ and 24 meters respectively from the TDR location. The TDR therefore displays a jump in reflected wave amplitude when it encounters a change in impedance along the conductor. This simulation was done based on the assumption that the total impedance of a segment of the conductor is concentrated at the end of thatsegment.

\section{LOAD INDENTIFICATION}

Different application-specific methods have been investigated to understand how the load affects the power consumption, power quality, efficiency, reactive power compensation, system planning, and so forth. At the core of these approaches are the needs to understand the characteristicload signature. Non-intrusive load monitoring (NILM) is one of the earliest approaches to nonintrusive monitoring and can determine the operating schedule of electrical loads in the target system from measurements made at a centralized location by identifying times in which the electrical power measurement changed from one nearly constant (steady- state) value to another, normally corresponding to loads either turning on or off and characterized by their magnitude and sign in real and reactive power. Reactive and active power is not unique and may become crowded and indistinguishable as the load number increases. Research similar to this includes echoresonance load monitoring (ERLM), sign-up load registration (SULR), and developing a library and taxonomy of load signatures [8-13]. The following steps are used to define the state and type of Loads. Fig. 9 shows a block diagram representing the load identification process.
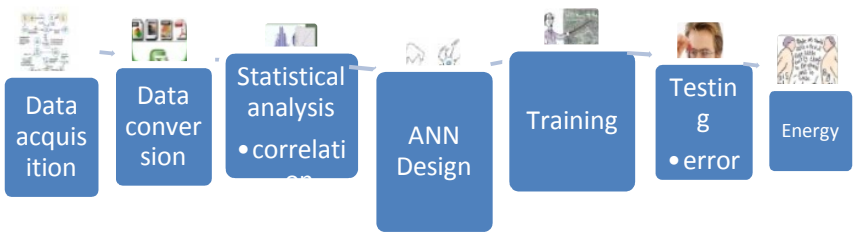

Figure 9, Steps used in the Load Prediction

\section{A. Defining the type of load (Load Recognition)}

In this section, a real-time power monitoring system is developed for data acquisition. A Current and Voltage transducer integrated with op amps and microcontroller will be installed in the panel. Using these power sensor current and voltage waveforms are sampled every 100 micro seconds, these samples (digital values) are sent to the computer via a serial port using the RS232 protocol. By using this process load characteristics will be measured and represented. A signal processing technique and estimation algorithm will be created for signal filtering, signal disaggregation, and load recognition. Previously, different methods have been used to understand the different load features (power quality, efficiency, reactive power compensation system planning, and load shedding). This approach tries to analyze the load signature. The concept of load recognition for the purposes of this paper is based on the special load signature in which every load has a unique feature in its consumption behavior that is very close to a human signature. Load features include the active and reactive power, current wave, and instantaneous power wave. The load disaggregation process is as follows:

- Data acquisition: First, the steady state and transient state signal will be captured. In the steady state, the fixed samples rate is set. The transient signal is used to capture the waveform during switching.

- Data Processing Model: The data from the signals will be conditioned, and re-sampled to align the current respective to the voltage and filtering.

- Event Detection: The data from the preceding steps will then be used to detect when the actual appliances are being switched on/off.

- Pattern Recognition: The artificial neural network (ANN) will be taught to learn the specific features of the different appliances and identify them. In this study, Multi-layer-Feed forward neural network is selected. The method used to train the system is based on the neural network approach [15].

- Committee Decision Mechanism: This mechanism will be used to extract different features such as the $\mathrm{PQ}$, current wave, harmonics, and the instantaneous power wave. This will be called the candidate pool. The committee Decision mechanism evaluates the potential solutions and renders the best final solution from a group of potential solutions [16].

\section{B. Detecting Load States}

The steady state, or the condition in which the phantom load exists and lots of energy is wasted, will be defined. The transient state and steady state determine what load type is turned "on". Therefore, the steady state plays a vital rule in terms of creating energy savings. The cluster analysis algorithm is used to locate these changes in two dimensional 
signature of $\Delta \mathrm{p}$ and $\Delta \mathrm{Q}$. The total load $\mathrm{P}[\mathrm{t}]$ is sampled at a fast sampling rate, and the difference in $\Delta \mathrm{P}[\mathrm{t}]$ between the two consecutive readings is calculated [17].

The appliance switches on/off as a result of the increase or decrease in the power level. In the first part of the study a bottom-up approach is used in which the majority of loads will be connected to sensors. As the study progresses, the configuration will be changed so that there will be one sensor in centralized location rather than on each node. The difference in the power level of the switching characteristics of the appliances is detected and calculated using the change in delta $\mathrm{P}$, given below:

$$
\Delta \mathrm{P}[\mathrm{t}]=\mathrm{P}[\mathrm{t}]-\mathrm{P}[\mathrm{t}-1]
$$

When it is difficult to determine the difference between $\Delta \mathrm{P}$ and $\Delta \mathrm{Q}$, another signature (like harmonics) can be used to define the loads[17].

\section{Neural Network}

The neural network being employed for this research is the Multi-Layer Feed Forward. It is also known as the multi-layer perception (MLP) network. This neural network consists of one or more hidden layers, whose computational nodes are correspondingly called hidden neurons. The purpose of the hidden neuron is to add up more computation between the input and output layer of cores in some useful manner. The input signal is applied to the neurons in the second layer. The output signal of second layer is used as inputs to the third layer, and so on for the rest of the network.

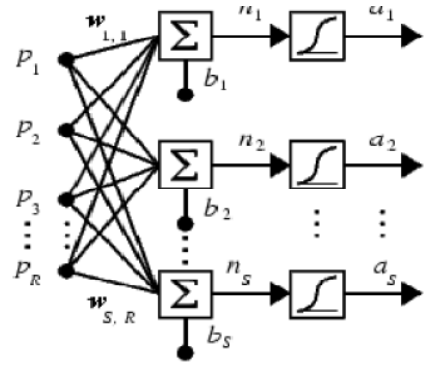

Figure 10: Topology of an MLP where R is the Number of Elements in the Input Vector, S is the Number of Neurons in the Layers, and Wij are the Weighting Factors between the Different Layers.

An MLP operates in two steps, training and testing. Training functions are used to minimize the difference between outputs of MLP and the desired values of the network. When the training state is done, neural weights will be frozen and new input data will be used to conclude the suitable output [18].

\section{1) Training and Testing pattern of Neural Network}

The training process uses a trial and error method to achieve the optimal behavior of the network. The fastest training function is trainlm, which is also the default training function for feed forward net. Other methods such as quasi-Newton method and trainbfg will also be explored to determine the best training of the network. After achieving, the optimal training, the network will be tested for load prediction accuracy. The current method under investigation is the ANN tool.

\section{2) Preliminary Results of Neural Network}

Each neural network represents one pattern (load). Some loads have the same signature and confusion of load identification could be avoided using more signatures like reactive power and harmonics. Decision tree system is used on the neural network system as a committee decision maker. Fig. 11 shows block diagram for pattern recognition in the Neural Network.

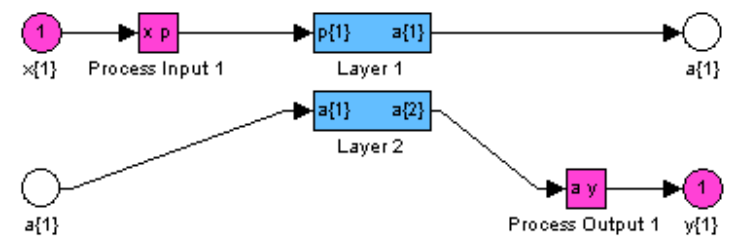

Figure 11, block diagram for Neural Network Simulation

Fig.12 shows pattern recognition for a sample load using the Neural Network shown in fig. 11.

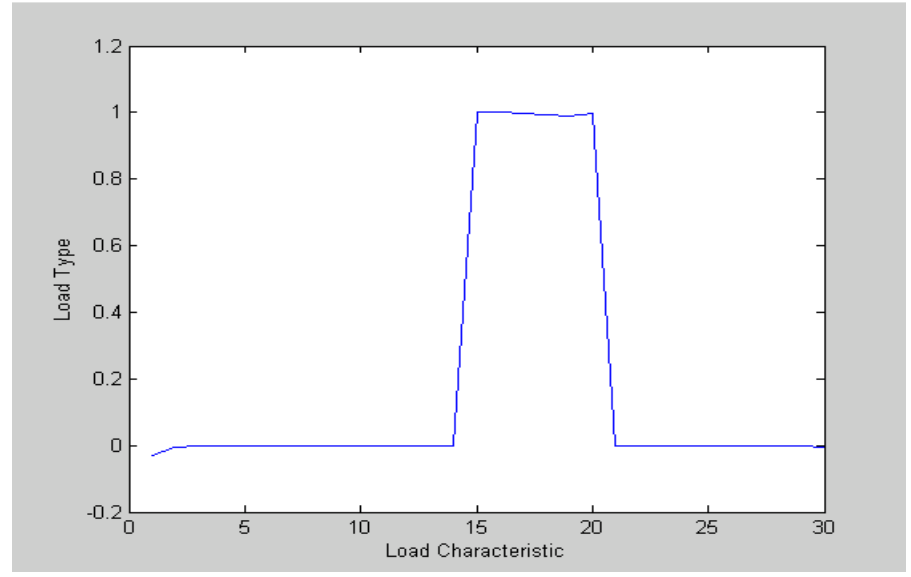

Figure 12, Load Type (Printer, Laptop...Etc.) vs. Load Characteristic (watt)

Defining phantom loads is the main purpose of the load type identification. The difference in power consumption for a specific loads will indicate whether the load is in the ON/OFF or Standby mode. With load location identified, the NILM will have additional information to accurately indentify the type of load.

\section{CONCLUSION}

In this paper, smart home technologies are being introduced to conserve energy in the built environment. The load location and identification methods by the TDR and NILM technologies will reduce hardware additions to existing electrical circuitry.

Preliminary results from the simulations indicate that a possible sensor technology can be developed to upgrade existing electrical systems. This will reduce cost of upgrading and increase energy efficiency in the built environment. 


\section{REFERENCES}

[1] U.S. Green Building Council, "Green Building Facts." U.S. Green Building Council. www.usgbc.org/ShowFile.aspx?DocumentID=5961, Accessed 7-21-2010.

[2] Chevron's energy efficiency and conservation, http://www.chevron.com/globalissues/energyefficiencyconservation/, Accessed 7-21-2010.

[3] Wisconsin Focus on Energy,"what is "phantom load" and how does it cost you money?" 2011.

[4] Ed Carroll, Eric Hatton, Mark Brown, "Residential Energy Use Behavior change Pilot," CMFS project code B21383, April 20, 2009 Franklin Energy.

[5] Karen Ehrhardt-Martinez, Kat A. Donnelly, \& John A. "Skip" Laitner, "Advanced metering Initiatives and Residential Feedback Programs: A Meta-Review of Household Electricity-Saving Opportunities," American Council for an Energy-Efficient Economy, report Number E105, June.

[6] Martin Hartebrodt et al. "Fault Detection in Fieldbuses with Time Domain Reflectometry", IEEE Africon 2004.

[7] Fawwaz T, Ulaby et al., Fundamentals of Applied Electromagnetics, $6^{\text {th }}$ ed, Prenice Hall, Boston, 2010.

[8] Shaw, S., S. Leeb, L. Norford and R. Cox, " Nonintrusive load monitoring and diagnostics in power systems," IEEE Trans. On Instrumentation and Measurement, Vol 57, issue 7, 2008, pp 1445-1454

[9] G. w. Hart, "Nonintrusive appliance load monitoring," Proc. Of the IEEE, Vol. 80, pp. 1870-1891. Dec, 1992.

[10] F. Sultanem, "Using appliance signatures for monitoring residential loads at meter panel level," IEEE Transactions on Power Delivery, Vol. 6, pp. 1380-1385, 1991.

[11] C. Laughman, K Lee, R. Cox, S. shaw, S. Leeb, L. Norford and P. Armstrong, "Power Signature analysis, "Power and Energy Magazine, IEEE, Vol. 1, pp. 56-63, 2003
[12] W K Lee, G S K Fung, H Y Lam, F H Y Chan, M. Lucente, “ Exploration on Load signatures", International conference on electrical Engineering (ICEE), July 2004, Japan.

[13] K. H. Ting, Mark Lucente, George S. K. Fung, W. K.. Lee and S. Y. R. Hui, "A taxonomy of Load Signatures for Single-Phase electric Appliances,” IEEE PESC, 12-18 June 2005, Brazil .

[14] Load signature study-part 1: basic concept, structure, and Methodology Jian Liang, Simon K. K, Member, IEEE, Gail Kendall, and John W. M. cheng, Member, IEEE.

[15] J. Duan et al. "Neural Network Approach for Estimating of Load comostion", 2004.

[16] Load signature Study-Part I: Basic Concept, Structure, and Methodology " Jian Liang, Simon K.k. Ng, Member, IEEE, Gail Kendall, and John W.M.Cheng", Member,IEEE

[17] Active power residential non-intrusive appliance load monitoring system "Arend j. bijker, xiaohua xia", senior member, IEEE, and jiangfeng zhang

[18] [20]-L.Fausett, Fundamentals of Neural Networks, prentice-Hall Inc., 1994.

[19] Guangming et al. (2008), "A Wireless Power Outlet System for Smart Homes" IEEE Transactions on Consumer Electronics, Vol. 54, No. 4.

[20] La Grou John. (2009). "John La Grou plugs smart power outlets". TED Conference.

[21] Lien et al. (2006). "Remotely Controllable Outlet System for Home Power Management" IEEE Tenth International Symposium on Consumer Electronics.

[22] National Fire Protection Association 70, National Electric Code-2011. 